\title{
Peso, talla y perímetro craneano de escolares de la Región Metropolitana de Chile, de acuerdo al nivel socioeconómico
}

\author{
Raquel Burrows A. ${ }^{1}$; Laura Leiva B. ${ }^{2}$; Santiago Muzzo B. \\ Weight, height and skull circumference of school age children from the \\ Metropolitan Region of Chile, according to socioeconomic level
}

\begin{abstract}
Body weight, stature and skull circumference were measured in a sample of 5557 school age children representative of the Metropolitan Region of Chile, aged 6 to 15 years. Socioeconomic condition was stratified by a modified Graffar score. Results were expressed according to age, sex and socioeconomic level. Lower weight and height were recorded among subjects of lower socioeconomic stratum from age six vear on. However, these differences dissapear along age in males, while persisting and even increasing until 14 years of age in females, spexiallv those of height. Skull circumference showed smaller differences arnong socioeconomic groups in both sexes, which also dissapear with age. These results show sexual dimorphym in height growth patterns. We cannot draw conclusion as to the influence of ethnic and environmental factors. These measurements are important for growth and development surveillance of children belonging to different socioeconomic levels, to describe secular trends of growth in our populations and to allow better critical appraisal and understanding of individual cases.
\end{abstract}

(Key words: Growth, height, weight, head circumference, socioeconom ic influences.)

La vigilancia periódica del peso, la talla y el perímetro craneano constituyen excelentes parámetros para evaluar el estado de salud y pesquisar en forma temprana alteraciones en el crecimiento y desarrollo. A nivel poblacional, permite conocer la tendencia secular del crecimiento. La estabilización del crecimiento estatural de una población traduce mejor expresión del potencial genético y desaparición de los factores ambientales que lo alteraban. Las poblaciones de países en desarrollo, en cambio, están incrementando su estatura, lo que sugiere que aún

1. Unidad de Endocrinología, Instituto de Nutrición y Tecrología de los Alimentos, Universidad de Chile.

2. Tecrólogo Médico. Unidad de Endocrinología, Instituto de Nutrición y Tecnología de los Alimentos, Universidad de Chile.

Fuente de financiamiento: FONDECYT, Proyecto 93/85. están superando factores ambientales que afectan el crecimiento'.

La influencia de factores genéticos y ambientales sobre el crecimiento explica las diferencias detectadas en el peso y la talla por niveles socioeconómicos (NSE) y por región, dentro de un mismo pais, en estudios realizados las últimas tres décadas ${ }^{2-11}$. En Chile se han demostra. do diferencias de crecimiento en preescolares y púberes de diferentes estratos socioeconómicos, atribuidas tanto a factores étnicos como ambientales $^{12-14}$ y posiblemente a diferencias en el grado de desarrollo puberal alcanzado, ya que la edad de desarrollo puberal parece influir más que la edad cronológica en el peso y la estatu$\operatorname{ra}^{2}, 3,15$.

Existen controversias acerca de cuáles serían las tablas ideales para evaluar el crecimiento. Se piensa que las curvas de países desarrollados son las adecuadas, por reflejar mejor el potencial genético de crecimiento de una población. Sin embargo, existen diferencias étnicas entre países y grupos poblacionales, que se traducen en diferencias del crecimiento y desarrollo ${ }^{2,} 3,16$. 
Esto indicaría que el uso de tablas de otros paises no es lo ideal para la evaluación de poblaciones con marcadas diferencias étnicas, ya que resultan ser poco sensibles. Esto ha llevado a postular la necesidad de confeccionar tablas que consideren no sólo el NSE, sino que además el origen étnico, el área geográfica, el promedio estatural de los padres, el tamaño de la familia y el grado de desarrollo puberal alcanzado, especialmente en la evaluación del caso individual ${ }^{4,15,17,18}$. Disponer de tablas nacionales separadas por NSE y actualizadas cada cierto número de años, permite observar la tendencia del crecimiento en los diferentes NSE y tener un instrumento más sensible para evaluar el caso individual que se comporta diferente a sus pares. En este estudio se registraron peso, talla y perímetro craneano de escolares chilenos de diferentes NSE, con el fin de actualizar la información existente sobre crecimiento pondoestatural en los distintos NSE y ofrecer elementos de juicio que permitan evaluar adecuadamente los casos individuales.

\section{Material y Método}

La muestra estuvo constituida por 5557 escolares de ambos sexos (2 816 mujeres entre 6 y 14 años 6 meses y 2741 varones entre 6 y 15 años 11 meses), representativos de educandos de esos tramos de edades de la educación fiscal, subvencionada $y$ particular, de la Región Metropolitana (RM) de Chile, la que tiene alrededor de $45 \%$ de la población escolar de Chile. La representatividad de la muestra por sexo, edad y tipo de establecimiento se estimó de acuerdo a los datos entregados por el Ministerio de Educación. De la lista de establecimientos educacionales (afio 1985) de la R.M. se sortearon los tres tipos de establecimientos (fiscal, subvencionado y particular), dándole a cada tipo la representatividad que le correspondia. Los alumnos de cada establecintiento se seleccionaron en forma aleatoria. El peso, la talla y et perímetro craneano fueron registrados por dos médicos pediatras, utilizando una balarza de precisión con cartabón incluido (Hispara ${ }^{(\mathrm{B})}$ ) y una huincha no distensible. El NSE se calificó según la escala de Graffar modificada 19 , que considera actividad y escolaridad del jefe de hogar $y$ caracteristicas de la vivienda y lo clasifica de 1 a 6 , desde el mayor al más bajo. Los escolares fueron ordenados en cuatro grupos: A, de los niveles socioeconómicos más altos (1 y 2); B, del nivel medio (3); C, del nivel medio bajo (4), y $D$, de los njveles bajo y miseria (5 y 6). Las diferencias estadísticas de los promedios fueron analizadas con la prueba $F$ (análisis de varianza que permite comparar los cuatro grupos), y por la de $t$ de Student (para comparar dos grupos), otorgando significación estadística a p menot de $5 \%$.

\section{Resultados}

La edad en meses y los promedios de peso, talla y perímetro craneano se presentan separa. dos por grupos etarios de 6 meses. La tabla 1 muestra el promedio estatural de las mujeres, por NSE. En todas las edades hubo diferencias significativas por nivel, siendo menores los promedios de estatura a menor NSE. Las diferencias del nivel alto (A) con el resto de los niveles (B, C y D) fueron esporádicas antes de los 10 años y persistentes después de esa edad. A los 14 años de edad las escolares de nivel alto tuvieron diferencias de 6,7 y 9 centímetros, respectivamente, en la estatura, con las niñas de niveles medio, medio-bajo y bajo. En todos los NSE, hubo una diferencia de 20 y $21 \mathrm{~cm}$ en Ja estatura, entre los 10 y los 14 años. La tabla 2 muestra los promedios de peso de las mujeres. El promedio de peso fue casi siempre mayor en las escolares de nivel alto, alcanzando diferencias significativas con el nivel bajo en casi todas las edades analizadas. Entre el nivel alto y los medios las diferencias fueron esporádicas y aparecieron sólo despuès de los 11 años, sien. do mayor el peso en las de nivel medio entre 12 y 13 años, y en las de nivel alto a los 14 años de edad. Entre los 10 y los 14 años la variación del promedio de peso fluctuó entre los $18 \mathrm{y}$ $20 \mathrm{~kg}$ en todos los NSE.

La tabla 3 muestra el promedio estatural de los varones. Hubo un menor promedio de talla a menor nivel socioeconómico a partir de los 6 años de edad. Al comparar los grupos, la diferencia fue significativa entre el nivel alto y el bajo hasta los 15 años y con el medio-bajo has. ta los 14 años de edad. Las diferencias entre el nivel alto y el medio se produjeron sólo entre los 13 y $\operatorname{los} 14$ años. Los escolares de nivel alto tuvieron diferencias en la estatura de 0,3 y $1,5 \mathrm{~cm}$, respectivamente, con los del nivel medio, medio. bajo y bajo, a los 15 años de edad. Entre los 12 y 15 años hubo una diferencia de estatura que fluctuó entre 17 y $25 \mathrm{~cm}$, correspondiendo la menor al nivel medio y la mayor al bajo. Con respecto at promedio de peso de los varones (tabla 4), hubo diferencias significativas por NSE entre los 6 y 14 años de edad. El nive] alto tuvo promedios de pesos significativamente mayores que los de los niveles medio-bajo y bajo hasta los 14 años, perdiéndose esta diferencia entre los 14 años 6 meses y los 15 años 11 meses. Los niveles alto y medio presentaron pro- 
Tabla I

Promedio de talla de mujeres de 6 a 16 años según nivel sacioeconómico

\begin{tabular}{|c|c|c|c|c|c|c|c|c|c|c|c|c|c|c|c|c|}
\hline \multirow[t]{2}{*}{$\begin{array}{l}\text { Edad } \\
\text { (meses) }\end{array}$} & \multicolumn{2}{|c|}{ (A) } & \multicolumn{5}{|c|}{$\begin{array}{l}\text { Nivel sociocconómico (1) } \\
\begin{array}{ll}\text { (B) } & \text { (C) }\end{array}\end{array}$} & \multicolumn{3}{|c|}{ (D) } & \multirow[t]{2}{*}{$\mathbf{p}^{\mathbf{a}}$} & \multicolumn{5}{|c|}{$\mathbf{p}^{b}$} \\
\hline & $\bar{x}$ & $\mathrm{DE} n$ & $\overline{\mathbf{x}}$ & $\mathrm{DE} \mathbf{n}$ & $\bar{x}$ & DE & $n$ & $\bar{x}$ & $\mathbf{D E}$ & n & & & & & & \\
\hline $72-77$ & 116,1 & $4,6 \quad 14$ & 1158 & 4,821 & 113,5 & 5.5 & 29 & 112,9 & 4,1 & 11 & 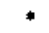 & $A / D^{*}$ & $\mathrm{~B} / \mathrm{D} *$ & & & \\
\hline $78-83$ & 117.3 & 4,116 & 118,0 & 4,921 & 116,7 & 5,2 & 43 & 114,6 & 3,9 & 19 & * & $\mathrm{A} / \mathrm{D}^{*}$ & $\mathrm{~B} / \mathrm{C} \cdot$ & $C / D^{*}$ & & \\
\hline 84-89 & 120,3 & 4,615 & 122,2 & 4,125 & 119,7 & 5,0 & 42 & 119,0 & 56 & 18 & $*$ & $\mathrm{~B} / \mathrm{C}$ & $\mathrm{B} / \mathrm{C} *$ & & & \\
\hline $90-95$ & 126,6 & 3.810 & 123,3 & 4426 & 121,7 & 5,1 & 36 & 121,7 & 5.9 & 19 & $*$ & $A / B *$ & $A / C^{* *}$ & & & \\
\hline $96-101$ & 125,3 & 3,311 & 126,6 & 5,137 & 124,9 & 4.2 & 43 & 122,8 & 6,3 & 21 & 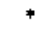 & $\mathrm{B} / \mathrm{D}$ * & & & & \\
\hline $102-107$ & 130,2 & 6,618 & 129.6 & 6,925 & 127,2 & 7.7 & 39 & 124,8 & 6,0 & 25 & $*$ & $\mathrm{~A} / \mathrm{D}^{*}$ & $\mathrm{~B} / \mathrm{D} *$ & & & \\
\hline $108-113$ & 127.6 & 4,112 & 1328 & 4,618 & 130,2 & 6,7 & 37 & 126,7 & 5,7 & 19 & $*+$ & $\mathrm{A} / \mathrm{B} * *$ & $\mathbf{B} / \mathbf{D} * *$ & $\mathrm{C} / \mathrm{D}^{*}$ & & \\
\hline $114-119$ & 1 36,2 & 6.417 & 132.6 & 6,527 & 132,6 & 7,0 & 44 & 131,8 & 5,5 & 28 & * & $\mathrm{A} / \mathrm{B} *$ & $A / C^{*}$ & $\mathrm{~A} / \mathrm{D}$ * & & \\
\hline $120-125$ & 139,0 & 6,610 & 135,2 & 5,434 & 135,2 & 6,6 & 50 & 132.6 & $\$ 5$ & 19 & $*$ & $\mathrm{~A} / \mathrm{D}=*$ & & & & \\
\hline $126-131$ & 141,4 & $5,8 \quad 19$ & 139.7 & 7.240 & 139.8 & 6.7 & 48 & 136,8 & 7,9 & 26 & $\bullet$ & $\mathrm{A} / \mathrm{D}=$ & & & & \\
\hline $132-137$ & 147,8 & 8,440 & 1419 & 7,873 & 141,4 & 7.4 & 107 & 140.2 & 7,2 & 42 & $\Rightarrow *$ & $\mathrm{~A} / \mathrm{B} *$ & $A / C^{* *}$ & $A_{p} D^{* *}$ & & \\
\hline $138-143$ & 149.2 & 8.535 & 1469 & 6,559 & 144,1 & 6.6 & 105 & 142,8 & 7,4 & 40 & $\omega m$ & $A / C * *$ & $A / D * *$ & B/C $* *$ & $\mathrm{~B}, \mathrm{D} * *$ & \\
\hline $144-149$ & 151,3 & 6,030 & 1497 & 3,268 & 147,8 & 6,6 & 116 & 144,7 & 7,8 & 64 & $+*$ & $A C C^{*}$ & $\mathrm{~A} / \mathrm{D}$ & $\mathbf{B}^{\prime} \mathbf{C}^{*}$ & $\mathrm{~B}_{j}^{\prime} \mathrm{D} * *$ & $\mathrm{CIO} * *$ \\
\hline $150-155$ & 152,8 & 5,824 & 153,4 & 6,369 & 149,5 & 6.7 & 107 & 147.0 & 5.8 & 37 & $*$ & $\mathrm{~A} / \mathrm{C}^{+*}$ & $A / D^{* *}$ & $\mathrm{~B} / \mathrm{C}^{* *}$ & $\mathrm{~B} ; \mathrm{D} *$ & CiD* \\
\hline $156-161$ & 154,4 & 5.735 & 154,7 & 5,767 & 151,3 & 5,9 & 111 & 150,3 & 6.5 & 45 & $H+$ & $\mathrm{A} / \mathrm{C} * *$ & $\mathrm{~A} / \mathrm{D}{ }^{* *}$ & $B / C *$ & Bi, $* *$ & \\
\hline $162-167$ & 159,0 & 6.817 & 153.7 & 6,658 & 153,0 & 6.0 & 109 & 152.5 & 3.7 & 44 & $* *$ & $\mathrm{~A} / \mathrm{B} * *$ & $\mathrm{~A} / \mathrm{C}^{* *}$ & $\mathrm{~A} / \mathrm{D} * *$ & & \\
\hline $168 \cdot 173$ & 161,4 & $3,3 \quad 9$ & 155.0 & 5,127 & 153.7 & 5,2 & 48 & 152,7 & 5.8 & 34 & $* *$ & $A / B * *$ & $\mathrm{~A} / \mathrm{C} * *$ & $A / D *$ & & \\
\hline
\end{tabular}

$\overline{\bar{x}}=$ Promedjo.

DE = Desviación estándar

n = Número de cosos

a $=$ Significancia según prueba $F$

b = Signifeanc1a según t de Student.

* = Significancia menor a 0,005 y mayor de 0.01.

$*$ * Significancia menor de 0,01 .

(1) = Vet categorias en el texto.

Tabla 2

Promedio de peso de mujeres de 6 a 16 años según nivel socioeconómico

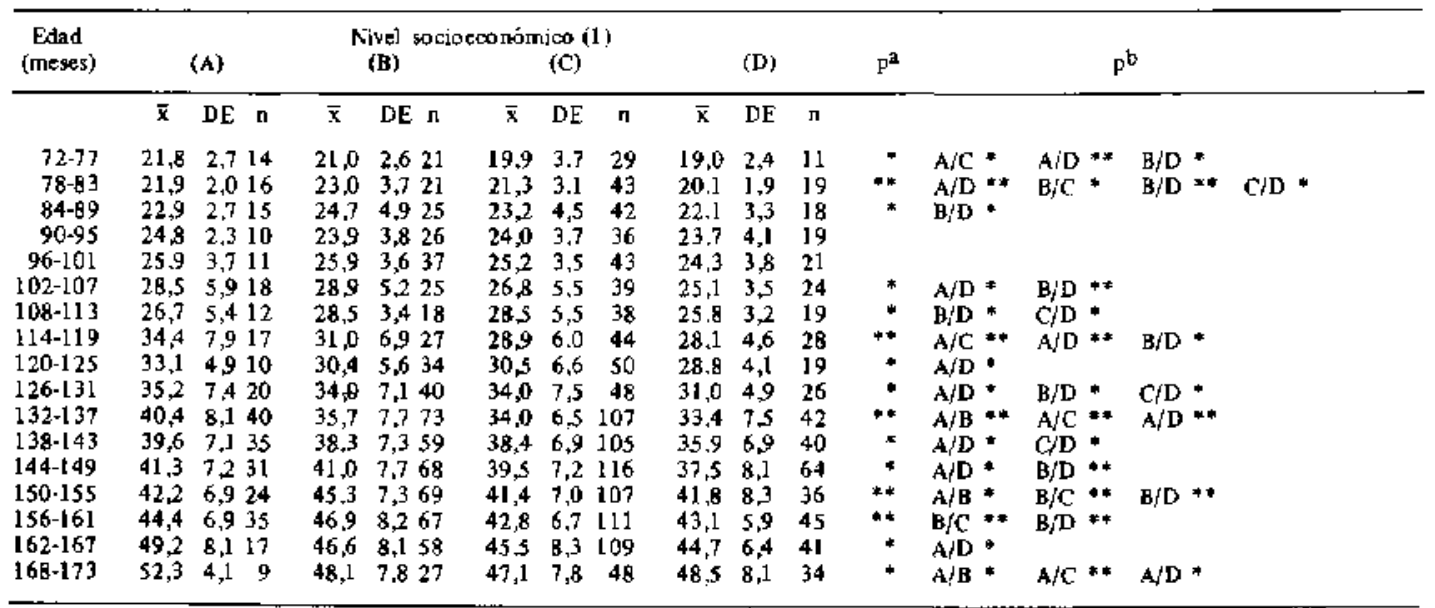

$\overline{\mathbf{x}}=$ Promadio

$\mathrm{DE}=$ Desviación estándar.

n $=$ Núrneto de casos

a = Significancia seqún prueba $F$

b = Significancia según t de Student.

- = Significancia menor a 0,05 y mayor de 0,01.

-* = Significancia menor de $0,0 \mathrm{I}$.

(1) = Ver categor ías en el texto. 
Tabla 3

Promedio de talla de varones de 6 a 15 años según nivel socioeconómico

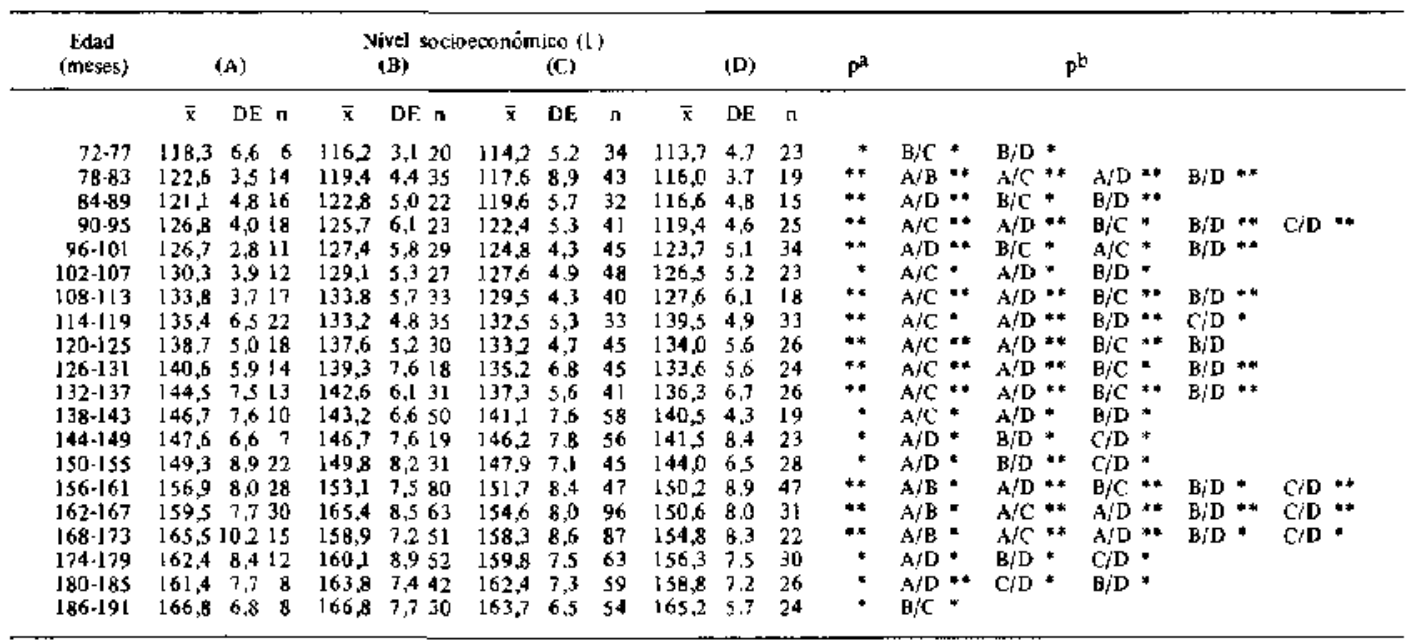

$\overline{\mathbf{\lambda}}=$ Promedio

$\mathrm{DE}=$ Desriación estándar.

n = Númoro de casos.

= Significancia scgun pructa 1

b = Significancia según t de Student.

* = Significancia menor a 0,05 y mayor de 0.01

= Significancia menorde0.01.

(1) = Ver categorías col texto.

Tabla 4

Promedio de peso de varones de 6 a 15 anos según nivel socioeconómico

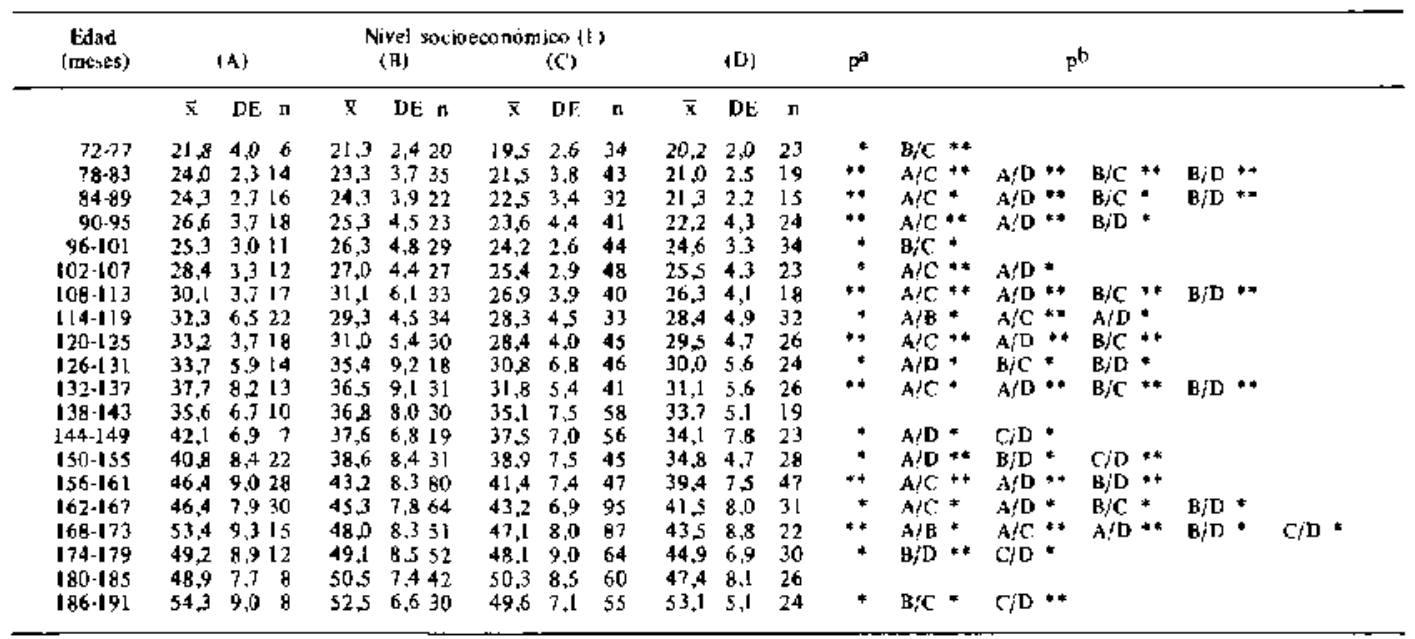

$\bar{x}=$ Promediu.

DE = Desviación estándar.

$n=$ Número de cavos,

a = Significancia según prueba $F$.

o Significancia según $\tau$ de Student.

- = Significancia menor a $0.05 y$ mayor de 0.01

* = Signifíancia menor de 0,01 .

(1) = Vur salcgor b́s en e] texto. 
medios de pesos similares, excepto en 2 grupos etarios (entre 9 años 6 meses y 9 años 11 meses y entre los 14 años y 14 años 5 meses), en que el nivel alto tuvo un promedio de peso signifi. cativamente mayor. Entre los 12 y los 15 años 11 meses hubo una diferencia en los promedios de peso que fluctuó entre los 12 y $\operatorname{los} 19 \mathrm{~kg}$, correspondiendo la menor al nivel alto $y$ la mayor al bajo.

La tabla 5 muestra que el promedio de perí. metro craneano en las mujeres tendió a ser mayor en e] NSE alto. Entre los 8 y los 11 años de edad las diferencias fueron significativas al comparar el nivel alto con los otros niveles. Después de los 11 años no hubo una clara relación por NSE, ya que los perímetros craneanos de los escolares de los niveles medio y bajo fueron en algunos casos mayores, similares o menores que los del nivel alto. Los promedios de perímetro craneano en los varones (tabla 6) mostraron diferencias por NSE a partir de los 6 años, con tendencia a desaparecer después de los 14 años. El njvel alto tuvo mayores promedios que el resto de los niveles sólo hasta los 13 anos de edad.

\section{Comentario}

Al comparar los promedios de peso, talla y circunferencia craneana de escolares de sexo masculino entre 6 y 15 años y de sexo femenino entre 6 y 14 años de la RM de Chile se encontra. ron diferencias significativas por NSE. Los promedios de estos parámetros fueron mayores a mayor NSE, pero las diferencias (excepto la estatura en las mujeres) tendieron a disminuir a desaparecer con la edad. Esto puede deberse a que, por tratarse de un estudio transversal, exista mayor selección en los escolares de los NSE más bajos (donde la deserción escolar es más prevalente) a mayor edad. En edades post. puberales las diferencias de estatura entre e] nivel alto y los otros njveles son mayores en las mujeres que en los varones. Este dimorfismo sexual ya ha sido demostrado en escolares de Santiago ${ }^{12}, 13,18$, atribuyéndolo a factores étnicos. En los varones, después de los 14 años, se observan distorsiones en los promedios de peso y talla, las que pueden deberse al reducido número de casos que constituyen cada muestra o a diferencias en el grado de desarrollo puberal alcanzado, el cual presenta mayor corre- lación con el peso y la talla, que la edad crono. lógica.

E] perímetro craneano fue la medición que mostró menores diferencias por NSE, $y$ en ambos sexos éstas fueron desaparecjendo con la edad. Esto se explicaría porque el estudio fue realizado en escolares que asisten a establecimientos para niños con rendimiento intelectual normal y porque en los escolares de menor NSE se produce una selección de los de mejor rendimiento, con la edad.

Se ha señalado que en la etapa prepuberal el crecimiento está influido especialmente por factores ambientales y en la pubertad por factores genéticos ${ }^{5}$. Si bien el dimorfismo sexual de la estatura sugiere la influencia de factores étnicos, no se puede descartar la influencia de factores ambientales en la menor estatura de los escolares de los NSE más bajos, al compararlos con el alto, pues afecta a ambos sexos y está presente desde edades tempranas. Los resultados descritos no nos permiten identificar factores etiológicos predominantes, ni evaluar por separado la influencia de los genéticos y los ambientales en el crecimiento y desarrollo de esta población de escolares. Sin embargo, poder contar con tablas separadas por NSE permite vigilar el crecimiento dentro de los diferentes njveles y a través de una evaluación periódica conocer la tendencia secular de nuestra población. Esto también contribuye a una mejor programación de medidas de acción de acuerdo a las variaciones detectadas en los distintos NSE. En casos individuales, el empleo de tablas separadas por NSE contribuye a un mejor análisis de niños que se comportan diferente a sus pares, ya que permite discriminar mejor grupos con problemas específicos de crecimiento y desarrollo dentro de una población.

\section{Resumen}

Se registraron el peso, la estatura y el perímetro craneano de 5557 escolares de ambos sexos, de 6 a 15 años de edad, en la Región Metropolitana. La condición socioeconómica se calificó mediante la escala de Graffar modificada, y los parámetros se expresaron como promedios, de acuerdo a ella, sexo y edad. En ambos sexos los promedios de peso y talla fueron menores cuando el nivel socioeconómico era más desfavorable. En los varones las diferencias entre grupos socioeconómicos disminuyeron con la edad; 
Tabla 5

Promedio de cráneo de mujeres de 6 a 16 años según nivel sociceconómico

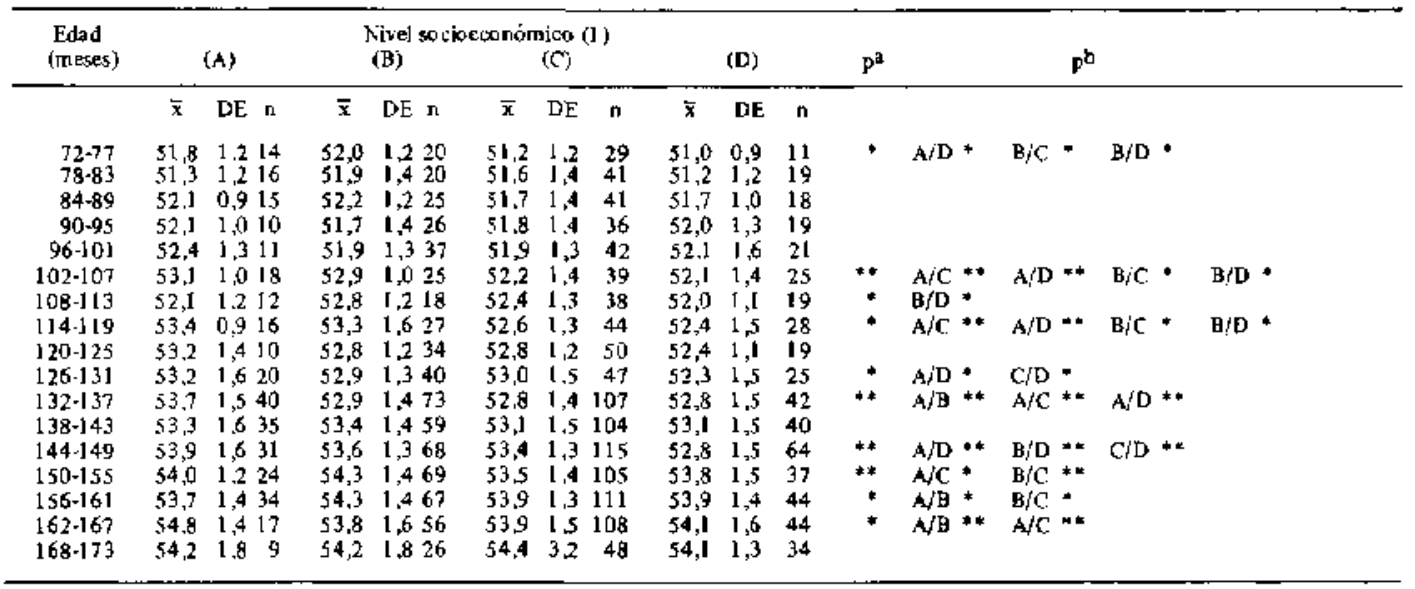

$\bar{x}=$ Promedio.

$\mathrm{DE}=$ Desviación estándar

n = Número de sasos.

a $=$ Significancia según prueba $\mathbf{F}$

b = Signifícancia según $t$ de Student.

- = Significanca menor a 0,0S y mayor de 0,01.

** = Significancia menor de 0,01 .

(1) = Ver categorías en el texto.

Tabla 6

Promedio de cráneo de varones de 6 a 15 años según nivel socioeconómico

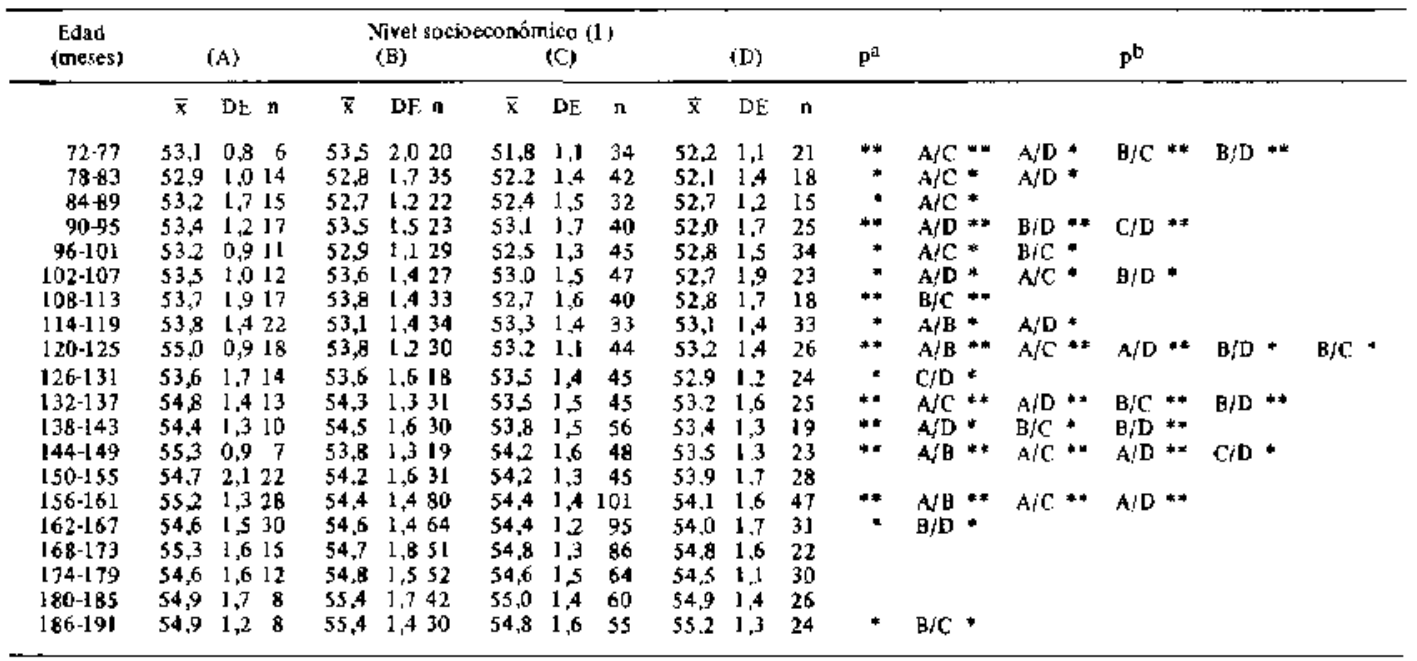

\footnotetext{
$\overrightarrow{\mathbf{x}}=$ Promedio.

DE = Desviación estándar.

$\mathrm{n}=$ Nímero de casos.

a = Significancia según prueba $F$.

b = Sanífieancia segúd t de Student.

* = Significancia menor a 0,05 y mayor de 0,01 .

$*$ = Significancia menor de 0,01

(1) = Ver categoris en el texto.
} 
en cambio en las mujeres persistieron, aumentando hasta los 14 años. El perímetro craneano mostró diferencias entre los distintos grupos, que fueron menos marcadas $y$ desaparecieron con la edad. Nuevamente se encontró el dimorfismo sexual descrito para la estatura, que ha sido atribuido a factores étnicos. Nuestros resultados no permiten diferenciar la influencia de factores genéticos y ambientales. Las tablas de crecimiento separadas por condición socio. económica permiten vigilar el crecimiento en los diferentes niveles, conocer su tendencia secular $y$, en casos individuales, estudiar mejor a los niños que se comportan diferente a sus pares.

(Palabras claves: Crecimiento, peso, estatura, perímetro craneano, nivel socioeconómico.)

\section{Referencias}

1. Smith WD: Basics and nature of growth. En: Smith WD ed. Growth and its Disorders, Phitadelphia: Saunders Company $1977 ; 1-17$.

2. Burrows $R$, Leiva $L$, Mauricci $A, Z$ vaighaft $A$, Muzzo $S$ : Caracter ísticas de la pubertad en escolares de la Región Metropolitana. Rev Chil Pediatr 1988; $59: 21-25$.

3. Muzzo $S$, Burrows $R, Z$ votghaft $A$ : Catacter ísticas de la pubertad de escolares de sexo masculino de diferentes niveles socioeconómicos de la Región Metropolitans de Chile. Rev Chil Pedjatr 1998; 59: 240-246.

4. Voss LD, Wilkin TJ. Berts PR: Do we need growth charts? Lancet $1987 ; 22: 447-448$.

5. Rona RJ: Genetic and environmental factors in the control of growth in childhood. Brit Med Bull $1981 ; 3: 265-272$.

6. Iyanovic $D$, Alvarez $M L$, Barrera $G$, Muzzo $S$ : Influencia del nivel socioeconómico en el estado nutricional de estudiantes egresados de educación básica y medir. Rev Med Chil 1984: 112: 1165-1171.

7. Lopez M. Tovar G, Ferid $N$. Esi udios comparados de la estatura y edad de la menarquia según estrato socioeconómico, en Venezuela, Arch Lat Nutr 1981;31: 740-757.

8. Beas $F$, Barrera $R$, Corvolín $L$, Cortinez A, Eggers $M$. Henriquez $C$ : Parámetios antropométricos en varones adolescentes chilenos de nivel socioeconómico medioelto. Rev Chil Pediatr 1986; 57: $485-490$

9. De la Fuente M, Margozzini JG: Antropometria de escolares de nivel socioeconómico alto. Estudio descriptivo. Rev Chil Pediatr $1983 ; 54: 353-357$.

10. Rona $R$, Pierret $T$ : Genotipo y estatura en nifios adolescentes de Santiago. Rev Med Chil 1973; 101: 207-211.

11. Youlton $R$, Valenzuela $C$ : Patrón de creciniento en estatura y peso de 0 a 17 años $y$ de circunferencia craneana de 0 a 2 años de nifios de estrato socioeconómico medio alto y alto de Santiago. Documento de la Rama de Endocrinología y Genética. 1990, Socieđad Chilena de Pediatría.

12. Volenzuela $C$, Avendaño A: Antropometría y maduración de escolares de un área de Santiago de Chile. Bol of Sanit Panam 1979;87; 113-131.

13. Muzzo $S$, Burnows $R$, Leiva $L$, Alvarez $M L$ : Características bjológicas, psicológicas y culturales dei adolescente chileno de diferentes NSE. Informe Técnico proyecto $93 / 85$ FONDECYT.

14. Barja I, De la Fuente $M$, Ballester $D$, Monckeberg $F$, Donoso $G$ : Peso y talla-de preescolares chilenos urbanos de tres niveles de vida. Rev Chil Pediatr $1965 ; 36: 525-529$.

15. Avendafo A, Volenzuela $C$, Huerta $J$, Gana $R$ : Crecimiento de varones y mujeres en etapa puberal. Rev Chil Pediatr 1989; 60: 255-261.

16. Valenzuela $C$ : Adelanto de la pubertad en escolares chilenos respecto a escolares europeos, medido por la velocidad de crecimiento estatural en un estudio transversal. Pediatría $1974 ; 20: 74-78$.

17. Chinn $S$, price $C E$, Rona $R J$ : Need for new reference curves for height. Afch Dis Child 1989; 64: $1545-1553$

18. Goldstein H, Tanner JM: Ecological considerations in the creation and the use of child growth creation and the use of child growth standards. Lancet $1980 ; 1: 582-585$.

19. Alvarez $M L$, Wurgaft $F$, Salazar $M$ : Mediciones del nivel socioeconómico bajo urbano en familias con lactantes desnutridos. Arch Latinoam Nutr 1982; 32: $650-662$. 